\title{
Sodium storage mechanism investigations through structural changes in hard carbons
}

\author{
Hande Alptekin, Heather Au, Anders Jensen, Emilia Olsson, \\ Mustafa Goktas, Thomas F Headen, Philipp Adelhelm, \\ Qiong Cai, Alan J Drew and Maria-Magdalena Titirici
}

\section{Published version information}

Citation: H Alptekin et al. 'Sodium storage mechanism investigations through structural changes in hard carbons.' ACS Applied Energy Materials, vol. 3, no. 10 (2020): acsaem.0c01614, 9918-9927.

\section{DOI: $10.1021 /$ acsaem.0c01614}

This document is the unedited author's version of a Submitted Work that was subsequently accepted for publication in ACS Applied Energy Materials, copyright (C)2020 American Chemical Society after peer review. To access the final edited and published work see DOI above.

Please cite only the published version using the reference above. This is the citation assigned by the publisher at the time of issuing the AAM. Please check the publisher's website for any updates. 


\section{Sodium Storage Mechanism Investigations Through Structural Changes in Hard Carbons}

Hande Alptekin, Heather Au, Anders Jensen, Emilia Olsson, Mustafa GOKTAS, Thomas F Headen, Philipp Adelhelm, Qiong Cai, Alan J Drew, and Maria-Magdalena Titirici

ACS Appl. Energy Mater., Just Accepted Manuscript • DOI: 10.1021/acsaem.0c01614 • Publication Date (Web): 18 Sep 2020

Downloaded from pubs.acs.org on September 28, 2020

\section{Just Accepted}

"Just Accepted" manuscripts have been peer-reviewed and accepted for publication. They are posted online prior to technical editing, formatting for publication and author proofing. The American Chemical Society provides "Just Accepted" as a service to the research community to expedite the dissemination of scientific material as soon as possible after acceptance. "Just Accepted" manuscripts appear in full in PDF format accompanied by an HTML abstract. "Just Accepted" manuscripts have been fully peer reviewed, but should not be considered the official version of record. They are citable by the Digital Object Identifier (DOI®). "Just Accepted" is an optional service offered to authors. Therefore, the "Just Accepted" Web site may not include all articles that will be published in the journal. After a manuscript is technically edited and formatted, it will be removed from the "Just Accepted" Web site and published as an ASAP article. Note that technical editing may introduce minor changes to the manuscript text and/or graphics which could affect content, and all legal disclaimers and ethical guidelines that apply to the journal pertain. ACS cannot be held responsible for errors or consequences arising from the use of information contained in these "Just Accepted" manuscripts. 


\title{
Sodium Storage Mechanism Investigations Through Structural Changes in Hard Carbons
}

\author{
Hande Alptekin ${ }^{1}$, Heather Au ${ }^{1}$, Anders CS Jensen ${ }^{1,2}$, Emilia Olsson ${ }^{1,2,3}$, Mustafa Goktas ${ }^{4}$, Thomas F. \\ Headen $^{5}$, Philipp Adelhelm ${ }^{4}$, Qiong $\mathrm{Cai}^{3}$, Alan J Drew ${ }^{2}$, Maria-Magdalena Titirici ${ }^{*}$ \\ ${ }^{1}$ Department of Chemical Engineering, Imperial College London, London SW7 2AZ, UK \\ ${ }^{2}$ School of Physics and Astronomy and Materials Science and Materials Research Institute, Queen \\ Mary University of London, London E1 4NS, UK \\ ${ }^{3}$ Department of Chemical and Process Engineering, University of Surrey, Guildford, GU2 7XH, \\ United Kingdom \\ ${ }^{4}$ Humboldt-University Berlin, Department of Chemistry, Brook-Taylor-Str. 2, D-12489 Berlin, \\ Germany \\ ${ }^{5}$ ISIS Pulsed Neutron and Muon Source, Rutherford Appleton Laboratory, STFC, Didcot OX11 0QX, \\ U.K. \\ *Corresponding author.Email: m.titirici@imperial.ac.uk
}

\begin{abstract}
Hard carbons, due to their relatively low cost and good electrochemical performance, are considered the most promising anode materials for Na-ion batteries. Despite the many reported structures of hard carbon, the practical use of hard carbon anodes is largely limited by low initial Coulombic efficiency (ICE) and the sodium storage mechanism still remains elusive. A better understanding of the sodium ion behaviour in hard carbon anodes is crucial to develop more efficient sodium ion batteries. Here, a series of hard carbon materials with tailored morphology and surface functionality was synthesized via hydrothermal carbonisation and subsequent pyrolysis from 1000 to $1900{ }^{\circ} \mathrm{C}$. Electrochemical results revealed different sodiation-desodiation trends in the galvanostatic potential profiles and varying ICE, and were compared with theoretical studies to understand the effect of the varying hard carbon structure on the sodium storage process at different voltages. Furthermore, electrode expansion during cycling was investigated by in-situ dilatometry; to the best of our knowledge, this is the first time the technique has been applied to hard carbons for ion storage mechanism investigation in Na-ion batteries. Combining experimental and theoretical results, we propose a model for sodium storage in our hard carbons that consists of Na-ion storage at defect sites and by intercalation in the high voltage slope region and via pore-filling in the low voltage plateau
\end{abstract}


region; these findings are important for the design of future electrode materials with high capacity and efficiency.

Keywords: hard carbon, sodium-ion batteries, storage mechanism, electrochemical dilatometry, green energy, sustainable materials.

\section{INTRODUCTION}

The growth in population and improved standard of living have resulted in an increased global energy consumption and is projected to account for an increase in energy demand of $90 \%$ by 2035. In light of this, the need to transform the way we produce energy has become a "must do now" operation. ${ }^{1-3}$ Renewable energy technologies such as solar, wind, and hydroelectric energy are considered to be the most sustainable energy sources, yet they are intermittent and the peak electrical production will not always match peak demand. Given the increased demand for cheap and efficient large-scale energy storage systems, the need to find an alternative to lithium-ion batteries (LIBs) is becoming urgent. Sodium-ion batteries (NIBs) have received significant attention as a potential eco-friendly and lower cost alternative to LIBs, owing to the abundance of sodium. ${ }^{1,4}$ Furthermore, cost benefits may also be achieved by the replacement of expensive copper foils, used as the current collector for LIB anodes, with inexpensive aluminium, and by replacing $\mathrm{Li}$ salts with $\mathrm{Na}$ salts in the electrolyte. ${ }^{5,6}$ Additionally, the conceptual similarity makes NIBs an attractive alternative to LIBs. ${ }^{7-9}$ Nevertheless, both technologies also show considerable differences that need to be overcome before NIBs can be a viable commercial alternative or complement to LIBs. One of the main limiting factors in transferring the LIB technology to NIBs is that graphite, the common LIB anode, cannot be applied as an anode material for NIBs. ${ }^{10,11}$ The limitation of graphite as a NIB anode material has hence led to alternative anode materials for NIBs being pursued. ${ }^{12,13}$ Recently, owing to their high sodium storage capacity, low working voltage, and excellent cycling stability, hard carbons with randomly oriented, disordered graphitic layers with "closed" micropores formed between these domains have been intensively studied as a NIB anode material, with promising performance being reported. ${ }^{14-16}$ These anodes can be derived from cheap and sustainable biosources, a key factor promoting their commercialisation for low cost and sustainable energy storage. ${ }^{14,17-21}$ Despite reports of numerous promising hard carbon materials, discrepancies in the understanding of sodium storage mechanism still exist. ${ }^{15,22}$ The effect of the pyrolysis temperature on the structural evolution, electrochemical performance and storage mechanism of the hard carbon has been extensively investigated. ${ }^{18,23,24}$ As a common trend, simultaneous 
decrease in surface area and the number of defects in hard carbon, and an increased degree of graphitization were observed with an increase in heat treatment temperature. All these morphological differences also create different electrode architecture and dynamics, which have considerable influence on sodium ion storage mechanism and the electrode's behaviour during battery cycling. In order to investigate the dynamic behaviour of the batteries, several in situ techniques have already been applied successfully, either on the cell, electrode or material level during the electrochemical cycling. One possible technique that allows indirectly to reveal the dynamic behaviour of crystalline anodes at the atomic level is in-situ X-ray diffraction (XRD) ${ }^{25-27}$ Also, in-situ Atomic Force Microscopy (AFM) can be used to study the volume change of active electrode particles during the electrochemical cycle. ${ }^{28,29}$ The most suitable method to investigate the dynamic changes of electrodes during an electrochemical cycle is in-situ electrochemical dilatometry (ECD). ${ }^{30-32}$ In the characteristic charge-discharge potential profiles, two distinct voltage regions have been observed; a sloping region above $0.1 \mathrm{~V}$, and a plateau below $0.1 \mathrm{~V}$. In earlier studies, these two distinct regions are assigned to different sodium storage mechanisms. The first model reported by Stevens and Dahn[17] assigned the sloping region to sodium ion insertion between the graphitic layers, and the plateau region to sodium filling or plating into pores. Later, Komaba et al. ${ }^{15}$ supported this mechanism by using ex-situ XRD to observe the expanded interlayer spacing because of sodium ion insertion, when hard carbon was discharged to $0.1 \mathrm{~V}$. Ex-situ small angle X-ray scattering (SAXS) analysis of the sample discharged below $0.2 \mathrm{~V}$ showed a decrease in the intensity of scattering from the pores, indicating that sodium ions inserted into the nanopores within this voltage range. Tarascon et al. ${ }^{33}$ tuned the microstructure of polyacrylonitrile (PAN)-derived carbon nanofibers (CNF) under various carbonization temperatures. By using X-ray photoelectron spectroscopy (XPS), temperature-programmed desorption coupled with mass spectrometry (TPD-MS) and gas adsorption techniques, the slope region was attributed to sodium ion adsorption on disordered graphitic sheets and the plateau region to the sodium ion filling into pores. The different mechanisms found in the literature may result from comparing carbon materials from different origins. Besides the type of materials, the applied experimental conditions and the characterization techniques used, and differences in detailed chemistry, especially heteroatomic doping, may be the cause of different conclusions.

In this paper, we synthesised a hard carbon series derived from hydrothermal treatment of glucose with citric acid as a catalyst (GCs) which were further carbonized at different temperatures. The resulting hard carbons exhibited a variety of textures and structures, all 
furthermore showing different electrode architectures with different degrees of surface functionality and defects, porosity and distinct electrochemical behaviour and Coulombic efficiencies. With the support of experimental and theoretical studies, modified structures were used to investigate the effect of hard carbon structure on the sodium storage mechanism. A fundamental understanding of the hard carbon morphology and the sodium storage mechanisms is essential for efficiently designed hard carbon materials for high capacity and high-efficiency NIBs.

\section{RESULTS AND DISCUSSION}

The SEM micrographs (Fig. S1) show that the GCs have a spherical shape with a relatively narrow particle size distribution, ranging from 5 to $8 \mu \mathrm{m}$. No change in particle size was observed upon pyrolysis, suggesting that the particle size is primarily determined by the Hydrothermal Carbonization (HTC) process which takes place at low temperatures (130-250 $\left.{ }^{\circ} \mathrm{C}\right) .{ }^{34}$ The coating process for electrode preparation did not change the GCs' material morphology and no change was observed in the particle size (Fig. S1f). Small angle neutron scattering (SANS) and small angle X-ray scattering (SAXS) ${ }^{35}$ were used to determine pore sizes. The pore size was modelled using a Guinier-Porod equation. ${ }^{35}$ SANS (Fig. 1a) and SAXS (Fig. S2) data show a Guinier plateau at around $0.1 \AA^{-1}$, indicating dilute nanopores of random size and shape (Fig. S2g). From this, the average pore size was determined (Fig. 1b) and showed a small increase from 1.5 to $2 \mathrm{~nm}$ between $1000^{\circ} \mathrm{C}$ to $1500^{\circ} \mathrm{C}$, followed by a sharper increase to $3.3 \mathrm{~nm}$ at $1900{ }^{\circ} \mathrm{C}$ (Fig. 1b). The pore size was also characterized by $\mathrm{N}_{2}$ and $\mathrm{CO}_{2}$ gas adsorption (Fig. S5). The $\mathrm{N}_{2}$ adsorption showed low surface area below $11 \mathrm{~m}^{2} / \mathrm{g}$ for all samples (Table 1), suggesting that there is little to no open porosity. The adsorption of $\mathrm{N}_{2}$ at $77 \mathrm{~K}$ has gas diffusion problems inside the narrowest micropores that prevent obtaining accurate micropore information, therefore it is widely used in analysis of mesopores. On the other hand, $\mathrm{CO}_{2}$ is more efficient at penetrating into the micropores due to its the higher saturation pressure (at $273 \mathrm{~K}$ as compared with nitrogen at $77 \mathrm{~K}$ ) and slightly smaller kinetic diameter. ${ }^{36}$ Therefore, $\mathrm{CO}_{2}$ gas adsorption of showed a much higher surface area ranging from 496 to $2.5 \mathrm{~m}^{2} / \mathrm{g}$ and a pore size range of $0.5-0.8 \mathrm{~nm}$ for all samples, significantly smaller than the pore sizes compared to the SAXS/SANS analysis. The $\mathrm{CO}_{2}$ adsorption surface area furthermore decreases with increasing temperature to just $2.6 \mathrm{~m}^{2} / \mathrm{g}$ for the high-temperature sample (GC1900). In SAXS, change in surface area can be analysed by the Guinier-Porod region $^{35}$ (Fig. S2h) which is proportional to the surface area of the nanopores. The intensity of the nano-pore feature in the SAXS/SANS measurements show a minor decrease with 
temperature suggesting that the pore-density remain largely constant (Fig. S2). This suggests that the gas adsorption measurements (Table 1) are either probing a surface structure which is removed at higher temperatures or the internal pores become more closed with increasing temperature as closed pores can be formed that are not accessible for gas probing but can be measured by X-ray and neutron scattering.
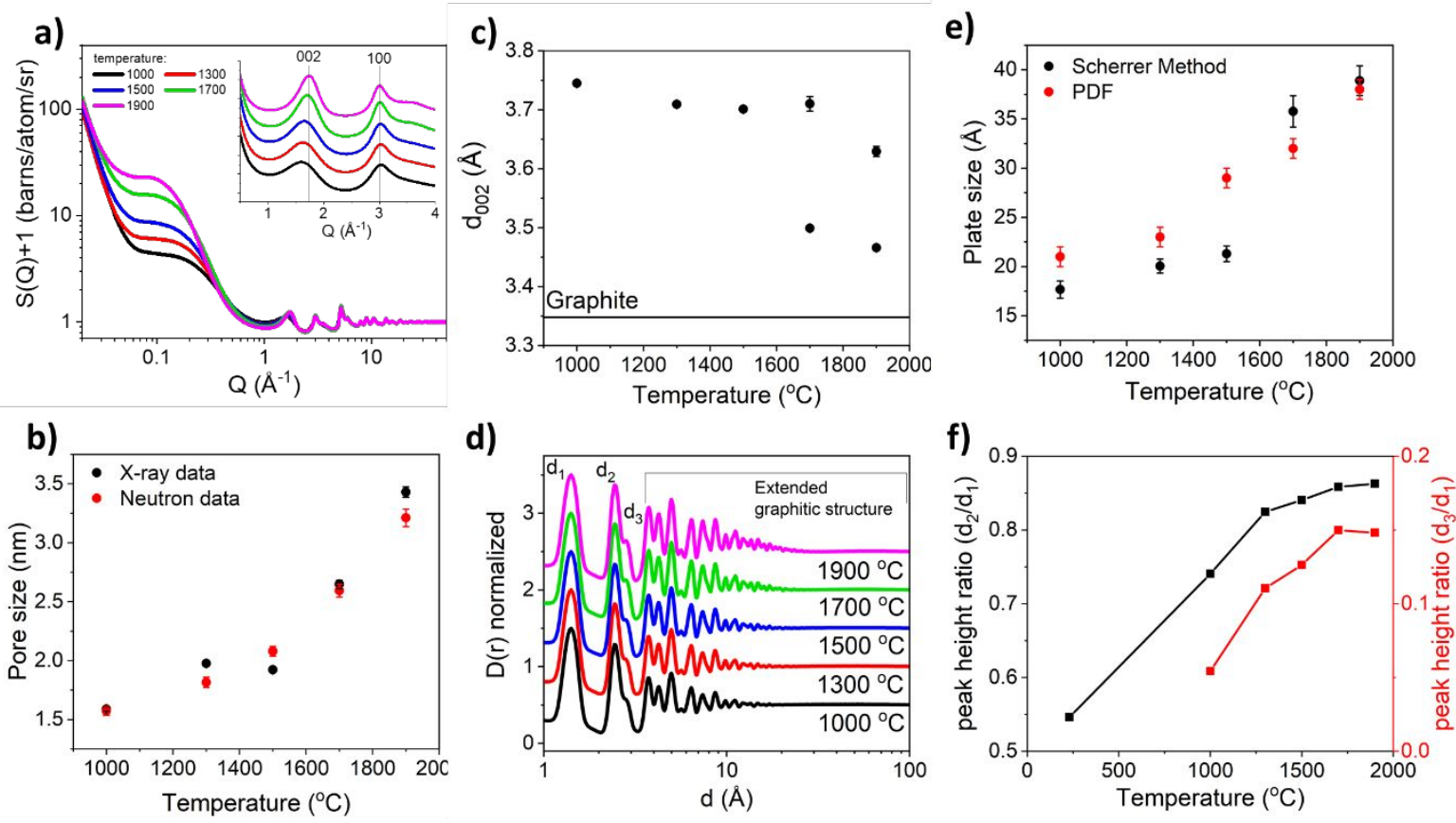

Fig. 1. a) Structure factor (The Guinier-Porod region showing scattering of the nanopores $\left(Q<1 \AA^{-1}\right)$ and scattering from the atomic structure at higher $Q$ ) and $b$ ) mean pore size determined from the GuinierPorod region of the SANS and SAXS data c) interlayer spacing of the graphitic d) planes pair distribution functions (PDF) of the GCs with the PDF normalized to the height of the first peak at $1.5 \AA$ e) size of the basal plane determined using the Scherrer method and the PDF, and f) peak height ratio of the first three peak observed in the PDF (Fig. 1d)

A graphitic local structure with turbostratic stacking of the basal plane is observed in the neutron total scattering (Fig. 1a) and WAXS measurements (Fig. S2). The data show the characteristic peaks from the plane of the (002) and (100) reflection at 1.8 and $3 \AA^{-1}$, respectively. The interlayer distances $\left(\mathrm{d}_{002}\right)$ (Fig. 1c) are well above the limit where sodium intercalation in graphitic layers is energetically favourable. ${ }^{37,38}$ At higher temperatures the 002 peak shifts to shorter distances and the peak shape becomes asymmetrical, requiring a two peak model to achieve a decent fit (Fig. S2e). This suggests that as the graphitic domains develop, some domains are not able to grow along the $c$-axis likely due to steric constraint. Therefore, a formation of two distinct populations in interlayer distance (Fig. 1c) are observed in the higher temperature materials. The mean size of the graphitic domains was determined by the Scherrer equation using the width of peak from the plane of the (100) reflection (Fig. 1a) and directly 
from the pair distribution functions (PDF) (Fig. 1d). Both techniques showed an increased domain size from $\sim 2 \mathrm{~nm}$ at $1000{ }^{\circ} \mathrm{C}$ to $\sim 4 \mathrm{~nm}$ at $1900{ }^{\circ} \mathrm{C}$ (Fig. 1e), which is an indication of structural ordering. The increase in the graphitic domain size is associated with a simultaneous Vincrease in the pore size, suggesting that the dimensions of the pores are determined by the size of the graphitic domains. The TEM images (Fig. 2) show that all samples have typically disordered carbon structures, although more organised regions with longer graphitic domains appear with the increase of the carbonisation temperature. Due to the presence of strong crosslinks formed during $\mathrm{HTC}$, even at $1900^{\circ} \mathrm{C}$, the material still shows the characteristics of hard carbon as the temperature was not sufficient to transform the structure to a highly ordered graphitic morphology. To further study the structural changes with carbonization temperature, Raman spectroscopy was conducted, and the results are shown in Fig. 2f, and Table 1. The Raman spectra exhibited two broad bands of the D band peak at $\sim 1340 \mathrm{~cm}^{-1}$ (the defect induced band) and $\mathrm{G}$ band peak at $\sim 1590 \mathrm{~cm}^{-1}$ (the crystalline graphite band). The D and G bands sharpen and become narrower with increasing carbonization temperature, which indicates the development of aromatic six-membered rings. The increase in the intensity ratio of D band over $\mathrm{G}$ band $\left(\mathrm{I}_{\mathrm{D}} / \mathrm{I}_{\mathrm{G}}\right)$ suggesting the increase in the concentration of defects along with the graphene sheets at higher carbonization temperatures. ${ }^{39}$ Moreover, the $L_{a}$ is calculated from the the $\mathrm{I}_{\mathrm{D}} / \mathrm{I}_{\mathrm{G}}$ ratio shows an increase in the crystallite length and coherence; and therefore a higher degree of graphitic order. ${ }^{40,41}$ Furthermore, the appearance of the $2 \mathrm{D}$ peak at 1700 and $1900^{\circ} \mathrm{C}$ implies the presence of larger domains of graphitic ordering, consistent with the neutron total scattering and SAXS/WAXS results and observations made by TEM. ${ }^{40-42}$ 

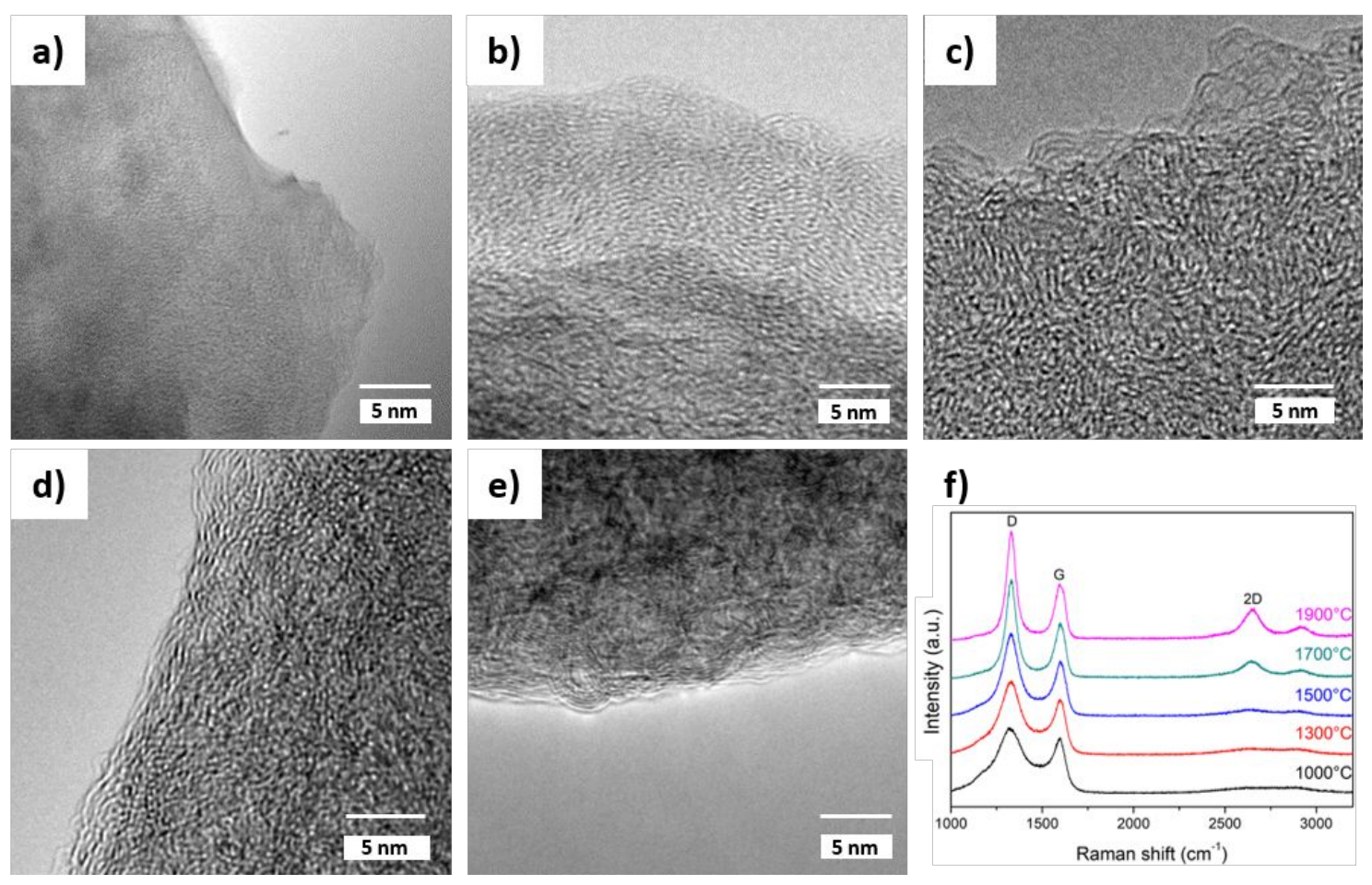

Fig. 2. TEM images of a) GC1000, b) GC1300, c) GC1500, d) GC1700, e) GC1900, and f) Raman spectra as a function of carbonization temperature.

The PDF can be used to observe changes in the local structure (Fig. 1d). The peak at $\sim 1.3 \AA$ (marked as $\mathrm{d}_{1}$ ) corresponds to the direct $\mathrm{C}-\mathrm{C}$ and $\mathrm{C}-\mathrm{O}$ bonds (a schematic of all the relevant distances is provided in Fig. S3 in the supporting information). The peak at $\sim 2.8 \AA$ (marked as $d_{2}$ ) relates to the second nearest neighbouring carbon and the presence of the signal at $\sim 3.1 \AA$ (marked as $\mathrm{d}_{3}$ ) indicates that the structure has the characteristic interatomic distance of the $\mathrm{C} 1$ C4 distance in a 6-membered aromatic ring structure. An increase in the ratio of the $d_{2} / d_{1}$ peak (Fig. 1f) shows that increasing carbonization temperature increases the average number of carbons in the $2^{\text {nd }}$ nearest neighbour position compared to the number of directly bonded carbons. The difference between the ratio of the $d_{2} / d_{1}$ is largest between the precursor (HTC sample before pyrolysis) and GC1000, as expected given that the HTC sample is dominated by 5-membered ring structures, aliphatic chains and contains few aromatic compounds. ${ }^{43}$ The difference between the $d_{2} / d_{1}$ ratios is also relatively large between GC1000 and GC1300. This difference decreases after GC1300, which shows that in between the graphitic domains there are still 5-membered ring structures, defects and crosslinkers left up to $1300^{\circ} \mathrm{C}$ that can act as feedstock for the formation of new graphitic domains, as the size of the graphitic domains does not change much in this temperature range. Above $1500^{\circ} \mathrm{C}$ the changes are likely related to the removal of edge terminations as the basal plane increases from 2 to $4 \mathrm{~nm}$ (Fig. 1e). This is supported by the increase in the $\mathrm{d}_{3} / \mathrm{d}_{1}$ intensity ratio, suggesting a higher density of aromatic 
structure with increasing carbonisation temperature. The presence of heteroatoms in the GCs was investigated by X-ray photoelectron spectroscopy (XPS) to evaluate concentration of heteroatoms that may contribute to possible Faradaic reactions occurring on the surface of the electrode material ${ }^{44,45}$, and the deconvoluted $\mathrm{C} 1 \mathrm{~s}$ and $\mathrm{O} 1 \mathrm{~s}$ spectra are shown in Fig. S5. The peaks at $533.3 \mathrm{eV}$ and $531.5 \mathrm{eV}$ in the $\mathrm{O} 1 \mathrm{~s}$ spectra are assigned to $\mathrm{C}-\mathrm{O}$ and $\mathrm{C}=\mathrm{O}$ species respectively. The proportion of these peaks decreases with increasing carbonization temperature, this assignment is confirmed by the deconvoluted peaks at $287.6 \mathrm{eV}$ and 288.2 $\mathrm{eV}$ in the $\mathrm{C} 1 \mathrm{~s}$ core-level spectrum. The XPS analysis (Table 1 and Fig. S5) shows that there is an increase in carbon content $(\mathrm{C} 1 \mathrm{~s}, \mathrm{at} \%)$ and a reduction in oxygen content $(\mathrm{O} 1 \mathrm{~s}, \mathrm{at} \%)$ with increasing carbonization temperature. Overall the high-temperature materials have fewer functional groups and defects, narrower interlayer distances and larger pore sizes compared to the low-temperature materials. ${ }^{46}$

Table 1. Summary of the characteristics of the GC materials. $\mathrm{S}_{\mathrm{N} 2}$ and $\mathrm{S}_{\mathrm{CO} 2}$ surface area determined by $\mathrm{N}_{2}$ and adsorption $\mathrm{CO}_{2}$ adsorption respectively. $\mathrm{C} 1 \mathrm{~s}$ and $\mathrm{O} 1 \mathrm{~s}$ are atomic compositions obtained by XPS.

\begin{tabular}{|c|c|c|c|c|c|}
\hline Materials & GC1000 & GC1300 & GC1500 & GC1700 & GC1900 \\
\hline $\mathrm{d}_{002}(\AA)$ & 3.75 & 3.68 & 3.66 & 3.58 & 3.43 \\
\hline $\mathrm{d}_{100}(\AA)$ & 2.04 & 2.04 & 2.04 & 2.05 & 2.10 \\
\hline Pore Size SAXS $(\mathrm{nm})$ & 1.6 & 2 & 2 & 2.6 & 3.5 \\
\hline $\mathrm{I}_{\mathrm{D}} / \mathrm{I}_{\mathrm{G}}$ & 1.15 & 1.32 & 1.50 & 1.82 & 2.02 \\
\hline $\mathrm{L}_{\mathrm{a}}(\mathrm{nm})$ & 0.9 & 1 & 1.1 & 1.2 & 1.3 \\
\hline $\mathrm{S}_{\mathrm{N} 2}\left(\mathrm{~m}^{2} \mathrm{~g}^{-1}\right)$ & 11 & 4 & 2 & 1 & 0.5 \\
\hline Pore Size $_{\mathrm{N} 2}(\mathrm{~nm})$ & 1.6 & 2.7 & 2.8 & 2.9 & 4.8 \\
\hline Pore Volume $_{\mathrm{N} 2}(\mathrm{cc} / \mathrm{g})$ & 0.021 & 0.020 & 0.018 & 0.003 & 0.003 \\
\hline $\mathrm{S}_{\mathrm{CO} 2}\left(\mathrm{~m}^{2} \mathrm{~g}^{-1}\right)$ & 496 & 164 & 76 & 5.2 & 2.6 \\
\hline Pore Volume $\mathrm{CO} 2(\mathrm{cc} / \mathrm{g})$ & 0.36 & 0.24 & 0.21 & 0.005 & 0.004 \\
\hline $\mathrm{C} 1 \mathrm{~s}, \%$ & 86.2 & 89.6 & 90.3 & 97.6 & 97.8 \\
\hline $\mathrm{O} 1 \mathrm{~s}, \%$ & 13.8 & 10.4 & 9.7 & 2.4 & 2.2 \\
\hline $1^{\text {st }}$ Cycle Capacity $\left(m A h \mathrm{~g}^{-1}\right)$ & 265 & 301 & 210 & 81 & 66 \\
\hline Initial Coulombic efficiency $\%$ & 72 & 83 & 74 & 76 & 66 \\
\hline
\end{tabular}

To observe the effects of all these structural differences on the electrochemical storage performance, GCs were electrochemically tested in half-cell configuration with Na metal as the counter electrode. Fig. S6 shows the first three cyclic voltammogram (CV) curves of the GCs at a scan rate of $0.1 \mathrm{mVs}^{-1}$. In the first cathodic scan of all the samples, the broad peak at around $0.4 \mathrm{~V}$ may be attributed to the reactions of the active sites on the carbon anode material with solvent molecules and electrolyte ions leading to the formation of the solid electrolyte interphase (SEI) in the first cycle. ${ }^{21}$ The irreversible area under the broad peak of GC1000 is much higher compared to the higher temperature materials, due to the higher number of defects 
and surface functional groups present which react irreversibly with the electrolyte. Additionally, GC1000 has a larger specific surface area which facilitates the SEI formation, and also contributes to the initial irreversible capacity. A sharp peak in the low potential region of $0.1 \mathrm{~V}$ can be ascribed to the oxidation or reduction reactions that involves electron transfer.

Fig. 3a shows the galvanostatic potential profiles of the first and second discharge-charge cycles for the hard carbons tested at $0.1 \mathrm{C}$. GC1300 delivers the highest total discharge capacity of $301 \mathrm{mAhg}^{-1}$ with an Initial Coulombic Efficiency (ICE) of 83\% (Table 1). The voltage profile can be divided into two regions, the high voltage sloping region between 1 and $0.1 \mathrm{~V}$ and the low voltage plateau region around $0.1 \mathrm{~V}$. The capacities of these separate regions were evaluated from the discharge profiles in the first cycles (Fig. 3b). It can be seen that as the carbonization temperature increases, the sloping region capacity contribution decreases. GC1000 with the highest oxygen content shows the largest sloping region capacity contribution (Fig. 3b), consistent with previously proposed sodium storage models where oxygencontaining defects offer favorable sodium adsorption sites. ${ }^{33,47}$ Besides the contribution of surface functional groups and defects, variations of interlayer distance $\left(\mathrm{d}_{002}\right)$ between graphitic layers also affect $\mathrm{Na}$ insertion, as the expanded graphitic layers serve as $\mathrm{Na}$ storage sites. A larger contribution to the sloping region is seen in the lower temperature hard carbons with larger interlayer distance as compared to the higher carbonization temperatures. Therefore, contribution of both $\mathrm{Na}$ adsorption on defect sites and insertion in turbostratic carbon domains can be observed in sloping voltage profiles. We assume that after all the existing interlayer spaces and defects reaches its maximum sodium storage capacity, pore filling starts corresponding to the low voltage plateau region. As in the example of temperature increase from $1000{ }^{\circ} \mathrm{C}$ to $1300{ }^{\circ} \mathrm{C}$, the plateau capacity of the higher temperature materials is expected to increase with the larger pore sizes. Nevertheless, at higher temperatures, while pore size continues to increase, the interlayer spacing narrows, the ion diffusion pathways to reach some pores therefore become inaccessible, corresponding to decrease in plateau region capacity. Therefore, an optimum concentration of internal (closed) porosity with energetically favorable interlayer distance is important to maximize the plateau capacity. 
a)

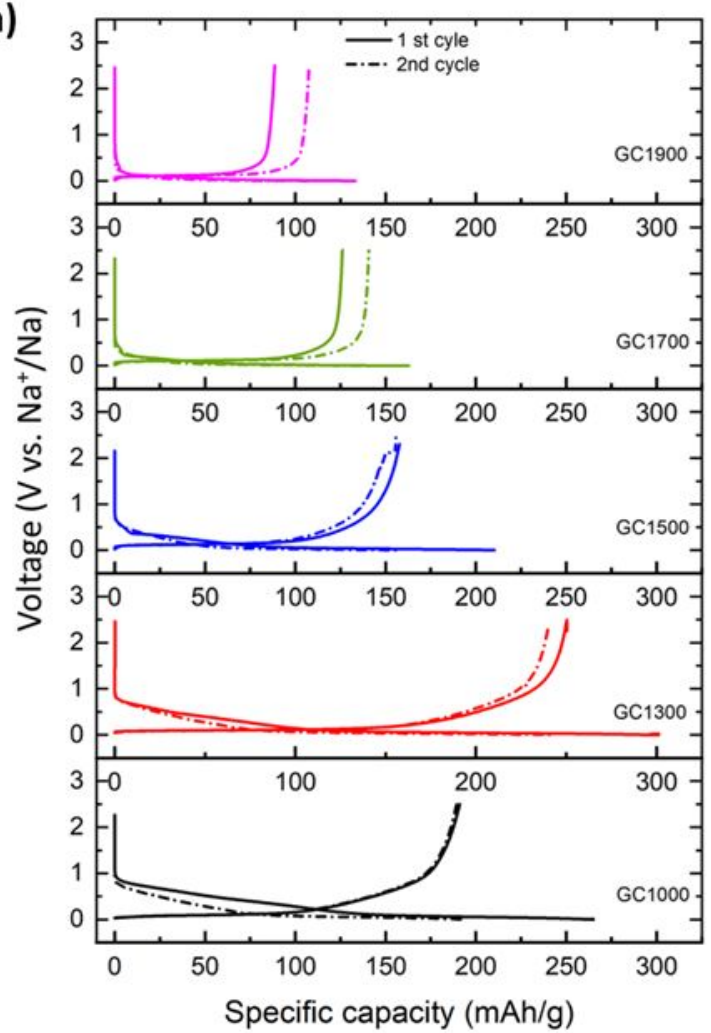

b)

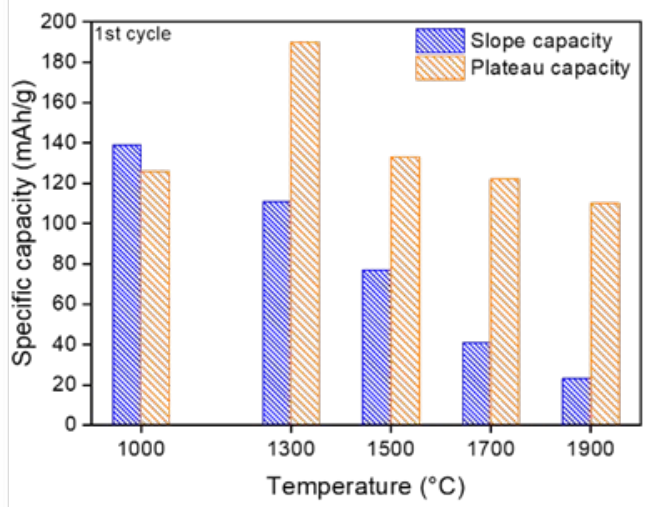

Fig. 3. a) The galvanostatic sodiation (discharge) and desodiation (charge) potential profiles of GC1000, GC1300, GC1500, GC1700 and GC1900. b) Specific capacities of different samples at high voltage sloping region $(0.1-1.0 \mathrm{~V}$ ) and low voltage plateau region (around $0.1 \mathrm{~V}$ )

The Na storage behaviour of the electrodes was further studied by in situ electrochemical dilatometry (ECD, Fig. S7a) over the first three cycles. The thickness of the electrodes used in dilatometry measurements meets (error margins are shown is Fig. S8) the thickness of electrodes used in the coin cell for the galvanostatic cycling. The initial thicknesses of GC1000, GC1300, GC1500, GC1700, GC1900 (film thickness without Al foil) were $104 \mu \mathrm{m}, 162 \mu \mathrm{m}$, $127 \mu \mathrm{m}, 94 \mu \mathrm{m}, 81 \mu \mathrm{m}$. As an example, Fig. 4a shows results for the carbon with the highest capacity (GC1300). Results for the other electrodes are shown in the supporting information, Fig. S8. Due to the difference of the cell setups of ECD and coin cells, ICE values (Table S1) and capacities obtained from each setup might be variable. As the loading and thickness of the different electrodes deviated from each other, it is difficult to establish a quantitative link between the performance of the electrode and their expansion/shrinkage. However, some interesting observations can be made. (1) The electrodes undergo an activation cycle, i.e. the first cycle leads to a slight increase in electrode thickness. This increase might originate from side reactions, e.g. SEI formation and/or rearrangement of particles that creates variation in 
electrode microstructure. (2)The expansion (difference in thickness between initial and desodiated state, Fig. S7) for all electrodes was in the range of 1.03 to $0.25 \%$ only (Table S1). These values are much smaller compared to when solvated sodium ions are intercalated ${ }^{31}$, indicating that solvent cointercalation into the carbon matrix does not take place. Small values can be also found for other carbon composites at similar capacity values. ${ }^{48,49}$ Considering full cells, minimal expansion will help to achieve a long cycle life.

\section{a)}

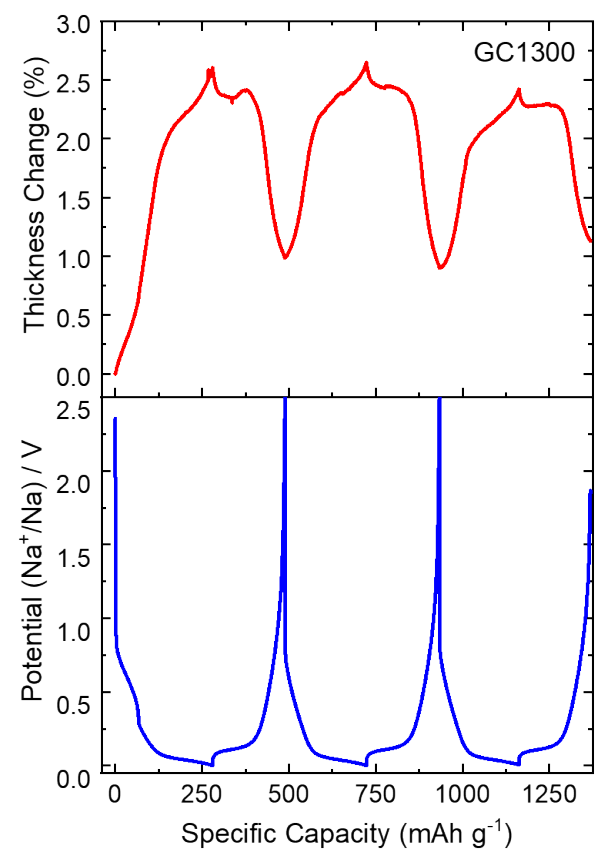

b)

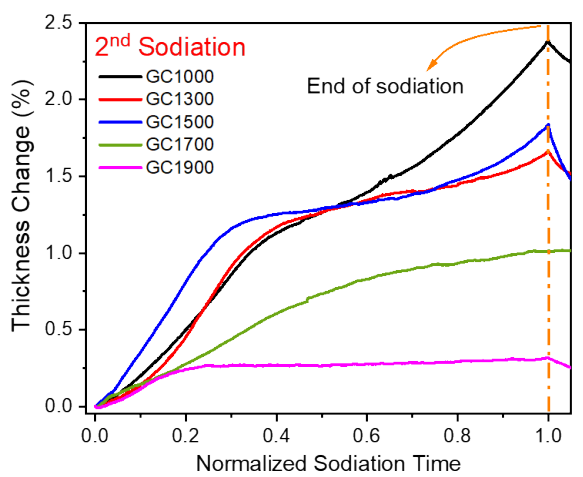

Fig. 4. a) In-situ electrochemical dilatometry measurements of the electrode thickness change inthe first three cycles for GC1300 b) percentage thickness change of each electrodes during the second sodiation.

(3) Fig. 4b compares the electrode expansion during sodiation for the different electrodes $\left(2^{\text {nd }}\right.$ cycle, data was normalized). Although sodiation occurs at constant current, the expansion of the electrode is not linear. As mentioned above, the voltage profile can be roughly divided into a sloping region and a plateau region. The ECD results show that the expansion of the electrode is greater in the sloping region than in the plateau region. This supports the concept that the sloping region is due to ion insertion between graphitic layers since sodium intercalation in graphite typically results in large interlayer expansion. ${ }^{50}$ Conversely, pore filling results in minimal thickness change, and therefore supports the assignment of this process occurring during the plateau region, where less pronounced electrode expansion is observed. The ex-situ SEM images of GC1300 (Fig. S9) show cross-sections of the electrode after the first sodiation and desodiation process. Since the electrode has a variable surface height, three different 
regions were measured to obtain the average thickness (before cycling $\sim 144$, after the first sodiation $\sim 178 \mu \mathrm{m}$, after the first cycle $\sim 170 \mu \mathrm{m}$ ). The result is consistent with the in-situ electrochemical dilatometry observation that the structure remained expanded as compared to the initial state of the electrode.

From the experimental characterisation of the hard carbon samples above, it was shown that the materials contain expanded graphitic stacks with different interlayer distances and graphitic surfaces with oxygen heteroatom defects. To bring atomistic insight into the effect of oxygencontaining surface defects and differing graphitic interlayer distances on the sodium storage and incorporation in the hard carbon samples, density functional theory (DFT) simulations were employed. The surface defects and oxygen heteroatoms on the hard carbon surfaces were modelled in terms of oxygen defects on a graphene monolayer. To understand the difference in intercalation behaviour for the different hard carbon samples with varying graphitic interlayer distance, a double layer planar graphitic model was employed. The effect of pore size on sodium incorporation in hard carbon was probed in a previous publication. ${ }^{51}$ To study the effect of different interlayer spacing on the sodium incorporation and migration, the sodium binding energy in the bi-layer graphitic layers with different $\mathrm{d}_{002}$ was calculated (see supporting information for details). To calculate the sodium binding energy $\left(E_{\text {bind }}\right)^{52,53}$, equation S2 was adapted to include sodium in between two graphitic sheets. The calculated binding energies are presented in Table $\mathrm{S} 2$, with a more negative $\mathrm{E}_{\text {bind }}$ indicating a more energetically stable structure. It is clearly seen from Table S2 that the sodium incorporation in the graphitic stacks become more favourable with increasing $\mathrm{d}_{002}$. Hence, GC1000 is the most favourable for sodium intercalation from these calculations, whereas GC1900 would not be expected to store much sodium in the graphitic stacks with calculated $\mathrm{d}_{002}$. The sloping region is partly attributed to sodium storage in expanded graphitic stacks. Hence, a stronger (more negative) $\mathrm{E}_{\mathrm{bind}}$ is in line with the more distinct sloping regions observed for the GC1000, GC1300, and GC1500 samples as compared to GC1900. From the calculated $\mathrm{E}_{\text {bind }}$, the Na storage capacity of GC1700 should also have contribution from the graphitic stack filling in its sloping region. However, the distinctly smaller sloping region of GC1700 compared to that of the lower carbonisation temperature samples (GC1000, GC1300, and GC1500) indicates that the capacity from the graphitic stack filling in GC1700 is relatively small. To form an understanding of the sodium storage in the graphitic models with different interlayer distances, the intercalation energy per sodium atom at different sodium loading (up to $8 \mathrm{Na}$ ) in the graphitic models of different interlayer distance (Fig. S10) was calculated. It is clearly seen that sodium intercalation in 
GC1900 is energetically unfavourable. GC1900 would hence be expected to have a lower sodium intercalation potential than the samples with higher average interlayer distance.

Overall, the calculated intercalation energies show that sodium intercalation increases with increasing the interlayer distance (Fig. S10), which could explain the higher sloping region capacities measured for the GC1000, GC1300 and GC1500 samples compared to high temperature materials GC1700 and GC1900. To assess the effect of oxygen-containing defects on sodium storage, which would contribute to the sloping region, a selection of graphene defects was modelled (Fig. S11). These defects have not been explicitly shown to be present in the experiments above, due to the difficulty in identifying/specifying the exact defects from the XPS analysis. Nonetheless, the models here allow us to explore different possible defect configurations at the atomic level and will serve as a guide to how oxygen-containing defects influence the sodium adsorption. In a previous publication, the effect of a wider selection of graphene defects on sodium adsorption was investigated, and reference to that study is made here. ${ }^{38}$ For each postulated defect in Fig. S11, its equilibrium defect formation energy $\left(E_{f}^{\text {defect }}\right)$ ${ }^{54,55}$ was calculated (see the supporting information). The calculated $E_{f}^{\text {defect }}$ are collected in Table S3 and show that oxygen-containing defects are energetically favourable on the graphene basal plane. Comparing $E_{f}^{\text {defect }}$, none of the investigated defects have higher $E_{f}^{\text {defect }}$ than the $3 \mathrm{O}_{\mathrm{C}}$ defect, or lower than the $2 \mathrm{O}_{\mathrm{C}}$ defect. The formation energies of the $\mathrm{O}_{\mathrm{C}}$ and $2 \mathrm{O}_{\mathrm{C}} \mathrm{V}_{\mathrm{C}}$ defects are furthermore relatively low, which indicates that a high concentration of these defects in the hard carbon basal planes would be probable. This confirms the experimental observation that hard carbons are prone to the formation of oxygen surface defects. To assess the sodium storage on these defective surfaces, the sodium adsorption energy $\left(E_{a d s}^{N a}\right)^{52,53}$ was calculated (equation S2). The more negative the adsorption energy is, the stronger the adsorption. The calculated adsorption energies for sodium on defect sites are collected in Table S2. The presence of oxygen-containing defects improves the sodium storage capacity of the carbon surface, except for the $\mathrm{O}_{\mathrm{C}}$ defect $(-2.85 \mathrm{eV})$ which shows no change in the sodium adsorption energy. The strong sodium adsorption energies calculated for the $\mathrm{O}_{\mathrm{C}} \mathrm{V}_{\mathrm{C}}$ defect indicate that this defect would greatly improve sodium storage. Hence, in the hard carbon samples with more oxygen content and oxygen surface defects (i.e. GC1000, GC1300, and GC1500), a considerable amount of sodium would be expected to be stored on defect sites. Therefore, a larger contribution to the sloping region capacity due to the presence of these kinds of defects in the lower temperature sample would be expected as compared to the GC1700 and GC1900 
samples. This is consistent with the XPS results in Table 1 which show that GC1700 and GC1900 have a lower oxygen content than the lower temperature samples, and hence the contribution from sodium storage on defect sites in the GC1700 and GC1900 samples will be much less. Hence, the sloping region of GC1700 is much shorter than for GC1000-1500.

\section{CONCLUSIONS}

In this study, a series of hard carbon materials derived from glucose was produced, and the effect of the different hard carbon configuration on the sodium storage mechanism investigated by using TEM, Raman, XPS, SAXS/WAXS, SANS, and gas adsorption techniques. It demonstrates that the presence of oxygen defects and large interlayer spacing has a more considerable contribution to the sloping region. Experimental results also supported by DFT simulations showing that sodium intercalation ability increases with increasing interlayer distance, while oxygen-containing surface defects improve the sloping region capacity. Increasing the number and size of the internal (closed) pores with increasing carbonization temperatures enhanced contribution of plateau region capacity up to $75 \%$ of total capacity. In situ ECD measurements reveal that expansion of the electrodes (expansion/shrinkage during cycling) is very small which is helpful considering full cells. Moreover, sodium storage during the sloping region leads to a greater electrode expansion compared to the plateau region. This led us to hypothesize that the slope region capacity is due to sodium ion adsorption on disordered graphene layers and intercalation into graphene layers with suitable spacing. In the plateau region sodium ion storage mechanism dominates over pore filling.

\section{EXPERIMENTAL METHODS}

Materials synthesis

Hard carbon materials used in this research were prepared from glucose in two steps that consist of hydrothermal carbonization at low temperature and carbonization at high temperature. ${ }^{34}$ In the first stage, $10 \mathrm{~g}$ glucose (D-(+)-glucose, $\geq 99.5 \%$, Sigma Aldrich) was dissolved is $90 \mathrm{ml}$ deionised water and the $\mathrm{pH}$ adjusted to $1-2$ by the addition of $3.6 \mathrm{~g}$ citric acid ( $\geq 99.5 \%$, Sigma Aldrich). The solution was placed in a Teflon container, then inside a stainless-steel autoclave vessel and heated at $230^{\circ} \mathrm{C}$ for $12 \mathrm{~h}$ then dried at $80{ }^{\circ} \mathrm{C}$ overnight. In the second stage, the resulting material was pyrolysed under continuous nitrogen gas flow in a tube furnace for $2 \mathrm{~h}$ with a heating rate of $5^{\circ} \mathrm{C} \mathrm{min}-1$. Pyrolysis was conducted at $1000^{\circ} \mathrm{C}, 1300^{\circ} \mathrm{C}, 1500^{\circ} \mathrm{C}, 1700^{\circ} \mathrm{C}$ and $1900{ }^{\circ} \mathrm{C}$. The resulting hard carbon samples are denoted GC1000, GC1300, GC1500, GC1700, and GC1900. 


\section{Materials characterization}

The morphology and structure of the samples were characterized by FEI INSPECT F Scanning Electron Microscope (SEM). Samples after cycling were washed with dimethyl carbonate and dried at $25{ }^{\circ} \mathrm{C}$ under vacuum overnight. For cross section measurements, electrodes were cut by scalpel. Transferring time for air sensitive sodiated samples was $60 \mathrm{~s}$ at maximum. Transmission electron microscopy (TEM) analysis was carried out by JEOL 2100Plus TEM at $200 \mathrm{kV}$ operating voltage. The textural properties of the GCs were determined from the adsorption isotherms of $\mathrm{N}_{2}$ at $77 \mathrm{~K}$ and $\mathrm{CO}_{2}$ at $273 \mathrm{~K}$ using Autosorb iQ-C (Quantachrome Instruments, USA) instrument. The specific surface areas were calculated by BrunauerEmmett-Teller (BET) method for $\mathrm{N}_{2}$ or Dubinin-Radushkevich (DR) method for $\mathrm{CO}_{2}$. The BET surface area $\left(\mathrm{S}_{\mathrm{BET}}\right)$ was calculated in the relative pressure range of $0.05-0.3$ for $\mathrm{N}_{2}$ adsorption. The pore size distribution (PSD) was calculated using a non-local density functional theory (NLDFT) method for slit pores adapted for these materials. Before the gas adsorption analysis samples were degassed overnight under vacuum at $200{ }^{\circ} \mathrm{C}$. Raman spectra were measured using a Renishaw in Via Raman instrument with a laser wavelength of $633 \mathrm{~nm}$. X-ray photoelectron spectroscopy (XPS) was performed using a Thermo Scientific K-Alpha* instrument with a monochromated Al-K $\alpha$ X-ray source, after drying the samples at $120^{\circ} \mathrm{C}$ overnight. Atomic compositions were obtained from the averaged spectra taken from 3 areas for each sample. Small/wide angle X-ray scattering (SAXS/WAXS) was measured on a Xenocs nano-InXider with a $\mathrm{Cu}-\mathrm{k} \alpha$ source and a two-detector setup for combined SAXS/WAXS measurements. Powder samples were packed in $2 \mathrm{~mm}$ Kapton capillaries and measured for 10 min, the empty capillary was measured separately and used for background subtraction. Neutron total scattering experiments were performed at the NIMROD ${ }^{56}$ instrument at ISIS (Harwell, UK), each sample was contained in a thin-walled Vanadium flat plate cell measured for 2 hours using a $30 \times 30 \mathrm{~mm}$ beam. The data were normalised using a $3 \mathrm{~mm} \mathrm{VNb}$ null scattering plate and scattering from the empty cell and instrument subtracted. The PDF and $\mathrm{S}(\mathrm{Q})$ were calculated using the Gudrun programme, both functions were normalised to absolute scale and corrected for beam attenuation, multiple-scattering and inelasticity effects. ${ }^{57,58}$

\section{Electrochemical measurements:}

Hard carbon materials coated on current collector were used as working electrode, and electrodes are prepared in the same way for all electrochemical measurements. Electrodes were made using an 85:5:10 weight ratio of active material, carbon black (Super P conductive, 99+\%, Alfa Aesar), and carboxymethyl cellulose binder (CMC) (Mw 250,000, Sigma 
Aldrich), respectively. The obtained slurry was coated in a $250 \mu \mathrm{m}$ layer on Al foil (conductive carbon coated, $18 \mu \mathrm{m}$, MTI corporation) by doctor blade and dried at room temperature for $24 \mathrm{~h}$ and then at $100^{\circ} \mathrm{C}$ for $2 \mathrm{~h}$ under vacuum. The electrolyte and half-cell preparation were conducted in an argon-filled glove box with $\mathrm{H}_{2} \mathrm{O}<0.5 \mathrm{ppm}, \mathrm{O}_{2}<0.5 \mathrm{ppm}$. The CR2032 coin cells were assembled with a sodium metal disc (1 mm thick, sodium ingot, 99.8\% metals basis, Alfa Aesar) as a counter electrode (CE), and a hard carbon anode as the working electrode (WE). The loading of electrode active materials on current collector was ranging from 2.5-5 $\mathrm{mg} / \mathrm{cm}^{2}$. Whatman $\mathrm{GF} / \mathrm{B}$ glass microfibre was used as a separator. A $1 \mathrm{M}$ electrolyte solution containing pre-dried $\left(80{ }^{\circ} \mathrm{C}\right.$ overnight) sodium hexafluorophosphate $\left(\mathrm{NaPF}_{6}\right.$ Alfa Aesar, $99+\%$ ) in predried (in $4 \AA$ porous molecular sieves, overnight) 1:1 volume mixture of ethylene carbonate (EC, anhydrous 99\%, Sigma Aldrich) and dimethyl carbonate (DMC, 99.9+\%, Sigma Aldrich) was prepared by stirring until fully dissolved. Galvanostatic cycling tests were performed on a Basytec system in the potential range of $0.001-2.5 \mathrm{~V} v s \mathrm{Na}^{+} / \mathrm{Na}$. Sodiation/desodiation current rate was $30 \mathrm{~mA} \mathrm{~g}^{-1}$ which corresponds to a cycling rate of $\mathrm{C} / 10$. Electrochemical dilatometry (ECD-3-nano cell by EL-CELL GmbH) was employed to study the electrode dynamics during cycling. ECD-nano cells are designed in a way that only quantifies the dilation of the working electrode in one dimension by the help of a sensor during the electrochemical cycling. The tests were performed in a three electrode-cell configuration having $10 \mathrm{~mm}$ diameter of electrode (here hard carbon, the loading of electrode active materials on current collector was ranging from $2-4.5 \mathrm{mg} / \mathrm{cm}^{2}$ ) as the working electrode and of sodium metal as the counter (12 mm diameter) and reference electrodes. A special ceramic porous separator, which was soaked in $400 \mu \mathrm{L}$ of electrolyte to wet the electrode, was fixed in position between a metal frame and the spring. Therefore, only the dilatation of the electrode was recorded. The cells were cycled galvanostatically at $10 \mathrm{~mA} \mathrm{~g}^{-1}$ at constant temperature $\left(25^{\circ} \mathrm{C}\right)$ in a chamber.

\section{Computational Methods}

For the atomic scale simulations of Na storage on defective graphene surfaces and in graphitic stacks with different interlayer distances, all calculations were conducted using density functional theory (DFT) as implemented in the Vienna Ab initio Simulation Package (VASP, version 5.3.5). ${ }^{59-62}$ To simulate sodium storage and migration in the planar graphitic layers with varying interlayer distance, a $4 \times 4 \times 2$ supercell ( 64 atoms) was constructed, whereby up to eight sodium was added to each cell in between the two planar graphitic layers. The two planar graphitic layers were placed in an $\mathrm{AB}$ stacking mode, whilst varying the interlayer distance 
( $\mathrm{d}_{002}$ according to experimental characterisation). For the calculations of sodium on defective graphitic surfaces, we used graphene to simulate the hard carbon basal plane. Based on previous experience of modelling this kind of systems, an $8 \times 8$ supercell (without defects 72 carbon atoms) with a converged vacuum gap of $15 \AA$ was used. ${ }^{38}$ All calculations used the projector-augmented wave method (PAW) to describe the ion-electron interaction. ${ }^{63}$ The plane wave cut-off and k-space integrals were chosen so that the total energy was converged to 1 $\mathrm{meV} /$ atom. The kinetic energy cut-off was $800 \mathrm{eV}$, with a $5 \times 5 \times 2 \Gamma$-centred Monkhorst-Pack grid to sample the Brillouin zone for the graphitic systems and a $9 \times 9 \times 1 \Gamma$-centred MonkhorstPack grid for the graphene models. ${ }^{64}$ To express the interacting electron exchange-correlation energy, the generalized gradient approximation (GGA) with Perdew-Burke-Ernzerhof (PBE) 65,66 functionals were used (with electronic convergence criteria of $10^{-5} \mathrm{eV}$ and ionic convergence criteria of $\left.10^{-3} \mathrm{eV} \cdot \AA^{-1}\right)$. All calculations were performed spin-polarized and are periodic. Due to the large polarizability of the graphite and sodium, it is necessary to include dispersion corrections to accurately estimate the metal adsorption and binding energies, in addition to the graphitic interlayer binding energy. ${ }^{67,68} \mathrm{Here}$, the DFT-D3 method with BeckeJohnson damping by Grimme and co-workers was selected. ${ }^{69,70}$

\section{Acknowledgements}

The authors would like to thank the Engineering and Physical Sciences Research Council for funding (EP/R021554/1). H.Al would like to acknowledge the funding provided by the Republic of Turkey Ministry of National Education. E.O. would like to acknowledge the use of Athena at HPC Midlands+, which was funded by the EPSRC under grant EP/ P020232/1, and the Eureka HPC cluster at the University of Surrey. We are grateful to the UK Materials and Molecular Modelling Hub for computational resources, which is partially funded by EPSRC (EP/P020194/1).

\section{Author contributions}

H.Al designed the study and drafted the article. H.Al. and H.Au synthesised the hard carbon samples and conducted the electrochemical testing for the NIB cells. H.Al obtained the SEM, gas adsorption experiments and Raman data. H.Au and H.Al. conducted the XPS, and TEM experiments. A.C.S.J. conducted the SAXS/WAXS measurements and analysis. A.C.S.J. and T.F.H. performed the SANS/WANS measurements and analysis. E.O. performed the DFT simulations and analysis. M.G and H.Al conducted the in-situ electrochemical dilatometry 
experiment. H.Au, E.O., A.C.S.J., an M.G. revised and edited the manuscript. P.A., Q.C., A.J.D. and M.-M.T. provided supervision and funding, and revised and edited the final manuscript.

\section{Competing interests}

The authors declare no competing interests.

\section{Associated Content}

Supporting Information Available: Additional materials characterisation: SEM; SAXS, WAXS and PDF; gas adsorption analysis; XPS spectra. Additional electrochemical characterisation: $\mathrm{CV}$ and rate performance; electrochemical dilatometry and electrode thickness calculations. Additional DFT-calculated binding energies and discussion. 


\section{References}

$1 \quad$ Larcher, D.; Tarascon, J.-M. Towards greener and more sustainable batteries for electrical energy storage. Nat Chem 2015; 7: 19-29.

$2 \quad$ Lewis, N.S. Powering the Planet. MRS Bull 2007; 32: 808-820.

3 International Energy Agency Key World Energy Statistics http://www.iea.org/publications/freepublications/publication/ key_world_energy_stats-1.pdf. 2011.https://www.iea.org/publications/freepublications/publication/KeyWorld2017.pdf (accessed 16 Oct2019).

4 Grey, C.P.; Tarascon, J.M. Sustainability and in situ monitoring in battery development. Nat Mater 2017; 16: 45-56.

5 Saurel, D.; Orayech, B.; Xiao, B.; Carriazo, D.; Li, X. From Charge Storage Mechanism to Performance : A Roadmap toward High Specific Energy Sodium-Ion Batteries through Carbon Anode Optimization. 2018; 1703268: 1-33.

6 Hwang, J.-Y.; Myung, S.-T.; Sun, Y.-K. Sodium-ion batteries: present and future. Chem Soc Rev 2017; 46: 3529-3614.

7 Ponrouch, A.; Monti, D.; Boschin, A.; Steen, B.; Johansson, P.; Palacín, M.R. Non-aqueous electrolytes for sodium-ion batteries. J Mater Chem A 2015; 3: $22-42$.

8 Li, L.; Zheng, Y.; Zhang, S.; Yang, J.; Shao, Z.; Guo, Z. Recent progress on sodium ion batteries: potential high-performance anodes. Energy Environ Sci 2018; 11: 2310-2340.

9 Li, Y.; Hu, Y.-S.S.; Titirici, M.-M.M.; Chen, L.; Huang, X. Hard Carbon Microtubes Made from Renewable Cotton as High-Performance Anode Material for Sodium-Ion Batteries. $A d v$ Energy Mater 2016; 6: 10-12.

10 Ge, P.; Fouletier, M. Electrochemical intercalation of sodium in graphite. Solid State Ionics 1988; 28-30: 1172-1175.

11 Metrot, A.; Guerard, D.; Billaud, D.; Herold, A. New results about the sodium-graphite system. Synth Met 1980; 1: 363-369.

12 Luo, W.; Shen, F.; Bommier, C.; Zhu, H.; Ji, X.; Hu, L. Na-Ion Battery Anodes: Materials and Electrochemistry. Acc Chem Res 2016; 49: 231-240.

13 Li, Y.; Lu, Y.; Zhao, C.; Hu, Y.S.; Titirici, M.M.; Li, H.; Huang, X.; Chen, L. Recent advances of electrode materials for low-cost sodium-ion batteries towards practical application for grid energy storage. Energy Storage Mater 2017; 7: 130-151.

14 Stevens, D.A.; Dahn, J.R. High Capacity Anode Materials for Rechargeable Sodium-Ion Batteries. J Electrochem Soc 2000; 147: 1271.

15 Komaba, S.; Murata, W.; Ishikawa, T.; Yabuuchi, N.; Ozeki, T.; Nakayama, T.; Ogata, A.; Gotoh, K.; Fujiwara, K. Electrochemical Na Insertion and Solid Electrolyte Interphase for Hard-Carbon Electrodes and Application to Na-Ion Batteries. Adv Funct Mater 2011; 21: 3859-3867.

16 Irisarri, E.; Ponrouch, A.; Palacin, M.R. Review-Hard Carbon Negative Electrode Materials for Sodium-Ion Batteries. J Electrochem Soc 2015; 162: A2476-A2482.

17 Li, Y.; Mu, L.; Hu, Y.-S.S.; Li, H.; Chen, L.; Huang, X. Pitch-derived amorphous carbon as high performance anode for sodium-ion batteries. Energy Storage Mater 2016; 2: 139-145.

18 Hou, H.; Qiu, X.; Wei, W.; Zhang, Y.; Ji, X. Carbon Anode Materials for Advanced SodiumIon Batteries. 2017.

19 Alcántara, R.; Lavela, P.; Ortiz, G.F.; Tirado, J.L. Carbon Microspheres Obtained from Resorcinol-Formaldehyde as High-Capacity Electrodes for Sodium-Ion Batteries. Electrochem Solid-State Lett 2005; 8: A222.

20 Bommier, C.; Mitlin, D.; Ji, X. Internal structure - Na storage mechanisms - Electrochemical performance relations in carbons. Prog Mater Sci 2018; 97: 170-203.

21 Stevens, D.A.; Dahn, J.R. The Mechanisms of Lithium and Sodium Insertion in Carbon Materials. J Electrochem Soc 2001; 148: A803.

22 Dahbi, M.; Yabuuchi, N.; Kubota, K.; Tokiwa, K.; Komaba, S. Negative electrodes for Na-ion batteries. Phys Chem Chem Phys 2014; 16: 15007.

23 Luo, W.; Schardt, J.; Bommier, C.; Wang, B.; Razink, J.; Simonsen, J.; Ji, X. Carbon 
nanofibers derived from cellulose nanofibers as a long-life anode material for rechargeable sodium-ion batteries. J Mater Chem A 2013; 1: 10662-10666.

24 Qiu, S.; Xiao, L.; Sushko, M.L.; Han, K.S.; Shao, Y.; Yan, M.; Liang, X.; Mai, L.; Feng, J.; Cao, Y.; Ai, X.; Yang, H.; Liu, J. Manipulating Adsorption-Insertion Mechanisms in Nanostructured Carbon Materials for High-Efficiency Sodium Ion Storage. Adv Energy Mater 2017; 7: 1700403.

25 Morcrettea, M.; Chabrec, Y.; Vaughand, G.; Amatuccib, G.; Lerichea, J.B.; Patouxa, S.; Masqueliera, C.; Tarascona, J.M. In situ X-ray diffraction techniques as a powerful tool to study battery electrode materials. Electrochim Acta 2002; 47.19: 3137-3149.

26 Placke, T.; Schmuelling, G.; Kloepsch, R.; Meister, P.; Fromm, O.; Hilbig, P.; Meyer, H.-W.; Winter, M. In situ X-ray Diffraction Studies of Cation and Anion Inter-calation into Graphitic Carbons for Electrochemical Energy Storage Applications. Zeitschrift für Anorg und Allg Chemie 2014; 640: 1996-2006.

27 Whitehead, A.; Edström, K.; Rao, N.; Sources, J.O. In situ X-ray diffraction studies of a graphite-based Li-ion battery negative electrode. J Power Sources 1996; 63.1: 41-45.

28 Han, M.; Zhu, C.; Ma, T.; Pan, Z.; Tao, Z.; Chen, J. In situ atomic force microscopy study of nano-micro sodium deposition in ester-based electrolytes. Chem Commun 2018; 54: 23812384.

29 Lacey, S.D.; Wan, J.; Cresce, A.V.W.; Russell, S.M.; Dai, J.; Bao, W.; Xu, K.; Hu, L. Atomic force microscopy studies on molybdenum disulfide flakes as sodium-ion anodes. Nano Lett 2015; 15: 1018-1024.

30 Goktas, M.; Bolli, C.; Buchheim, J.; Berg, E.J.; Novák, P.; Bonilla, F.; Rojo, T.; Komaba, S.; Kubota, K.; Adelhelm, P. Stable and Unstable Diglyme-Based Electrolytes for Batteries with Sodium or Graphite as Electrode. 2019; 11: 32844-32855.

31 Goktas, M.; Bolli, C.; Berg, E.J.; Novák, P.; Pollok, K.; Langenhorst, F.; Roeder, M. v.; Lenchuk, O.; Mollenhauer, D.; Adelhelm, P. Graphite as Cointercalation Electrode for Sodium-Ion Batteries: Electrode Dynamics and the Missing Solid Electrolyte Interphase (SEI). Adv Energy Mater 2018; 8: 1702724.

32 Armand, M.; Touzain, P. Graphite intercalation compounds as cathode materials. Mater Sci Eng 1977; 31: 319-329.

33 Zhang, B.; Ghimbeu, C.M.; Laberty, C.; Vix-Guterl, C.; Tarascon, J.-M.M. Correlation Between Microstructure and Na Storage Behavior in Hard Carbon. Adv Energy Mater 2016; 6: 1501588.

34 Titirici, M.-M.; White, R.J.; Falco, C.; Sevilla, M. Black perspectives for a green future: hydrothermal carbons for environment protection and energy storage. Energy Environ Sci 2012; 5: 6796.

35 Stevens, D.A.; Dahn, J.R. An In Situ Small-Angle X-Ray Scattering Study of Sodium Insertion into a Nanoporous Carbon Anode Material within an Operating Electrochemical Cell. J Electrochem Soc 2000; 147: 4428.

36 Cychosz, K.A.; Guillet-Nicolas, R.; García-Martínez, J.; Thommes, M. Recent advances in the textural characterization of hierarchically structured nanoporous materials. Chem Soc Rev 2017; 46: 389-414.

37 Jensen, A.C.S.; Olsson, E.; Au, H.; Alptekin, H.; Yang, Z.; Cottrell, S.; Yokoyama, K.; Cai, Q.; Titirici, M.M.; Drew, A.J. Local mobility in electrochemically inactive sodium in hard carbon anodes after the first cycle. J Mater Chem A 2020; 8: 743-749.

38 Olsson, E.; Chai, G.; Dove, M.; Cai, Q. Adsorption and migration of alkali metals (Li, Na, and K) on pristine and defective graphene surfaces. Nanoscale 2019; 11: 5274-5284.

39 Ferrari, A.C.; John Robertson. Raman spectroscopy of amorphous, nanostructured, diamondlike carbon, and nanodiamond. Philos Trans R Soc London 2004; 362.1824: 2477-2512.

40 Ferrari, A.C.; Robertson, J. Interpretation of Raman spectra of disordered and amorphous carbon. Phys Rev B 2000; 61: 14095-14107.

41 Ferrari, A.C.; Basko, D.M. Raman spectroscopy as a versatile tool for studying the properties of graphene. Nat Nanotechnol 2013; 8: 235-246.

42 Ferrari, A.C.; Meyer, J.C.; Scardaci, V.; Casiraghi, C.; Lazzeri, M.; Mauri, F.; Piscanec, S.; 
Jiang, D.; Novoselov, K.S.; Roth, S.; Geim, A.K. Raman Spectrum of Graphene and Graphene Layers. Phys Rev Lett 2006; 97.18: 187401.

Titirici, M.-M.; Antonietti, M.; Baccile, N. Hydrothermal carbon from biomass: a comparison of the local structure from poly- to monosaccharides and pentoses/hexoses. Green Chem $\mathbf{2 0 0 8}$ 10: 1204

44 Augustyn, V.; Simon, P.; Dunn, B. Pseudocapacitive oxide materials for high-rate electrochemical energy storage. Energy Environ. Sci. 2014; 7: 1597-1614. Wang, J.; Polleux, J.; Lim, J.; Dunn, B. Pseudocapacitive contributions to electrochemical energy storage in TiO 2 (anatase) nanoparticles. J Phys Chem C 2007; 111: 14925-14931. on the $\mathrm{Na}+$ ion storage mechanism in hard carbon: Discrimination between the porosity, surface functional groups and defects. Nano Energy 2018; 44: 327-335.

Bommier, C.; Surta, T.W.; Dolgos, M.; Ji, X. New Mechanistic Insights on Na-Ion Storage in Nongraphitizable Carbon. Nano Lett 2015; 15: 5888-5892.

Gómez-Cámer, J.L.; Bünzli, C.; Hantel, M.M.; Poux, T.; Novák, P. On the correlation between electrode expansion and cycling stability of graphite/Si electrodes for Li-ion batteries. Carbon N Y 2016; 105: 42-51.

Palaniselvam, T.; Goktas, M.; Anothumakkool, B.; Sun, Y.N.; Schmuch, R.; Zhao, L.; Han, B.H.; Winter, M.; Adelhelm, P. Sodium Storage and Electrode Dynamics of Tin-Carbon Composite Electrodes from Bulk Precursors for Sodium-Ion Batteries. Adv Funct Mater 2019; 29: 1900790.

Au, H.; Rubio Carrero, N.; Buckley, D.J.; Mattevi, C.; Shaffer, M. Thermal decomposition of ternary sodium graphite intercalation compounds. Chem - A Eur J 2020; 26.29: 6545-6553. Bhide, A.; Hofmann, J.; Dü, A.K.; Rgen Janek, J.; Adelhelm, P. Electrochemical stability of non-aqueous electrolytes for sodium-ion batteries and their compatibility with $\mathrm{Na} 0.7 \mathrm{CoO} 2 \uparrow$. Phys Chem Chem Phys 2014; 16: 1987-1998.

Tsai, P.; Chung, S.-C.; Lin, S.; Yamada, A. Ab initio study of sodium intercalation into disordered carbon. J Mater Chem A 2015; 3: 9763-9768.

Nakada, K.; Ishii, A. Migration of adatom adsorption on graphene using DFT calculation. Solid State Commun 2011; 151: 13-16.

Raebiger, H.; Lany, S.; Zunger, A. Origins of the p-type nature and cation deficiency in $\mathrm{Cu} 2 \mathrm{O}$ and related materials. Phys Rev B 2007; 76: 045209.

Zhang, S.; Northrup, J. Chemical potential dependence of defect formation energies in GaAs: Application to Ga self-diffusion. Phys Rev Lett 1991; 67: 2339-2342.

Dove Martin. Local structure study of non-graphitisable carbon anode for sodium batteries. STFC ISIS Neutron Muon Source 2018. doi:10.5286/ISIS.E.RB1810728.

\section{Soper, A.K. Rutherford Appleton Laboratory Technical Report RAL-TR-2011-013. 2011.}

Keen, D.A. A comparison of various commonly used correlation functions for describing total scattering. J Appl Crystallogr 2001; 34: 172-177.

9 Kresse, G.; Furthmüller, J. Efficiency of ab-initio total energy calculations for metals and semiconductors using a plane-wave basis set. Comput Mater Sci 1996; 6: 15-50.

Kresse, G.; Furthmüller, J. Efficient iterative schemes for ab initio total-energy calculations using a plane-wave basis set. Phys Rev B Condens Matter 1996; 54: 11169-11186.

Kresse, G.; Hafner, J. Ab initio molecular-dynamics simulation of the liquid-metalamorphous-semiconductor transition in germanium. Phys Rev B 1994; 49: 14251.

62 Kresse, G.; Hafner, J. Ab initio molecular dynamics for liquid metals. Phys Rev B 1993; 47: 558.

Blöchl, P.E. Projector augmented-wave method. Phys Rev B 1994; 50: 17953.

Monkhorst, H.J.; Pack, J.D. Special points for Brillouin-zone integrations. Phys Rev B 1976; 13: 5188-5192.

65 Perdew, J.; Burke, K.; Ernzerhof, M. Errata: Generalized Gradient Approximation Made Simple. Phys Rev Lett 1997; 77: 1396.

66 Perdew, J.; Burke, K.; Ernzerhof, M. Generalized Gradient Approximation Made Simple. Phys Rev Lett 1996; 77: 3865-3868. 
67 Fan, X.; Zheng, W.T.; Kuo, J.-L.L.; Singh, D.J. Adsorption of single Li and the formation of small Li clusters on graphene for the anode of lithium-ion batteries. ACS Appl Mater Interfaces 2013; 5: 7793-7797.

68 Amft, M.; Lebègue, S.; Eriksson, O.; Skorodumova, N. V. Adsorption of Cu, Ag, and Au atoms on graphene including van der Waals interactions. J Phys Condens Matter 2011; 23: 395001.

69 Becke, A.D.; Johnson, E.R. Density-functional thermochemistry. III. The role of exact exchange. J Chem Phys 2005; 123: 154108.

70 Grimme, S.; Ehrlich, S.; Goerigk, L. Effect of the Damping Function in Dispersion Corrected Density Functional Theory. J Comput Chem 2011; 32: 1456. 
For Table of Contents Only

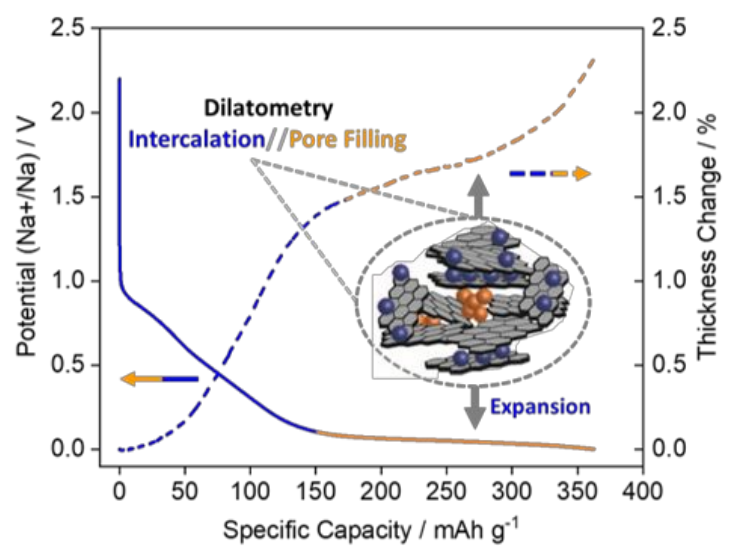

